\title{
Developing near-atomic-scale chemical analysis in liquid-phase S/TEM to study high capacity battery anodes
}

\author{
Rui Serra Maia ${ }^{1}$, Eric Detsi ${ }^{1}$, John Corsi ${ }^{2}$, Min Wang ${ }^{1}$, James Pikul ${ }^{3}$ and Eric Stach ${ }^{1}$ \\ ${ }^{1}$ University of Pennsylvania, Philadelphia, Pennsylvania, United States, ${ }^{2}$ University of Pennsylvania, \\ Philadelphia, United States, ${ }^{3}$ University of Pennsylvania, Philadeliphi, Pennsylvania, United States
}

The development of solutions to address the most significant world challenges, such as global energy supply and climate change, depends on our ability to study and understand fundamental physical and chemical processes in real-world conditions. For example, to achieve large-scale $\mathrm{CO} 2$ electrochemical reduction to liquid hydrocarbons, it is crucial to develop highly-selective catalysts with enhanced kinetics and operational stability.[1] However, thus far, it is not clear how the overall performance of the catalysts used in this process can be improved because most studies were only able to study the final reaction products and thus could not establish time-resolved property-activity relationships.

The development of liquid cells for in situ Scanning/Transmission electron microscopy created an entirely new field of research that enables tracking reaction systems in real-world conditions.[2] However, in liquid conditions, the thick layer of liquid that the electron beam must cross strongly degrades the beam coherence and energy resolution.[3] For that reason, obtaining chemical and structural analysis with the nanoscale resolution has been an unresolved problem during Scanning/Transmission Electron Microscopy (S/TEM) studies in a liquid environment. This has hindered the study of numerous liquid-phase nanoscale systems with world-wide scientific and technological importance, such as fuel-cells, batteries, and synthetic chemistry.[4,5]

We present a method to perform near atomic-scale chemical and structural analysis during liquid-phase Scanning/Transmission Electron microscopy. To overcome the detrimental effects of the electrolyte, we perform electrochemical water splitting in situ to generate a gas bubble that reduces the thickness of the liquid to a thin film that remains covering the sample (Fig. 1). The reduced thickness of liquid allows the acquisition of atomic-scale S/TEM images with chemical and valence analysis through Energy Dispersive Spectroscopy (EDS) and Electron Energy Loss Spectroscopy (EELS). This contrasts with a specimen cell completely filled with liquid, where intense electron beam scattering from the liquid obscures the EDS and EELS signal from the sample (Fig. 2). The generation of the gas bubble is fully reversible. This allows one to alternate between a full cell and thin-film condition, to obtain optimal experimental and analytical conditions. When the thin film is formed, we can reach near-atomic resolution EDS/EELS and obtain SAED data, both with excellent signal-to-noise ratios, while the sample remains in liquid conditions. Electrochemical studies also show that the thin film's electrochemical and ion transport properties are identical to those observed when the specimen cell is filled with electrolyte. This new method enables studying the chemical properties of materials at conditions they are subjected to during important industrial and technological applications.

The method developed here to obtain atomic-scale chemical analysis establishes a platform for understanding numerous other solid/liquid nanoscale systems with world-wide scientific and technological importance, such as fuel-cells, batteries, and synthetic chemistry. 




b Specimen cell is entirely filled with liquid

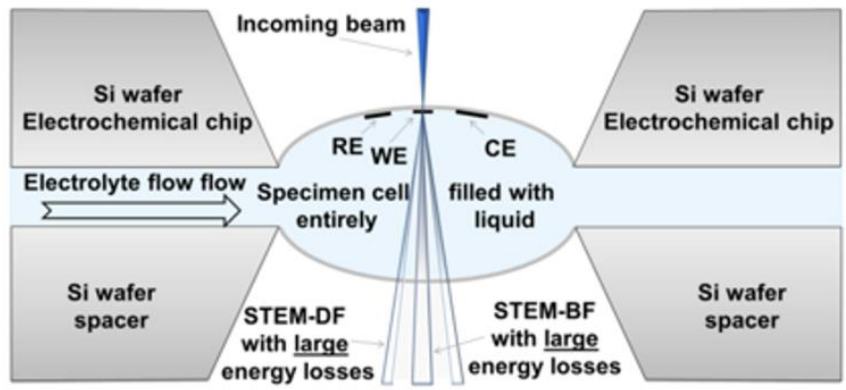

\section{c Thin-film of liquid remains covering the sample}



Figure 1. Figure 1. Liquid-phase TEM electrochemical cell used in this work. (a) Schematic of the chip equipped with active electrodes and the spacer. The chip is mounted on top of the spacer with the electrodes facing the spacer. The spacer contains raised posts that dictate the spacing between the two chips. For optimal resolution the sample should be deposited on the chip window or electrodes so that the STEM electron probe reaches the sample with its smallest diameter, before scattering through the liquid enclosed between the two windows. The red dashed line marks the cross-section of the schematic in (b) and (c). (b) Longwise vertical cross-section of liquid-phase in situ/operando TEM specimen chamber when the cell is entirely filled with liquid and (c) After Ewe $=2 \mathrm{~V}$ is held for $2 \mathrm{~s}$ to generate a gas bubble that reduces the liquid to a thin film that remains covering the windows, the electrodes and the sample. 


\section{Full liquid cell}


Figure 2. Figure 2. Energy dispersive spectroscopy (EDS) analysis of two partly de-alloyed AuAg samples. The EDS signal is obscured and produces uninterpretable results when the liquid cell is entirely filled with liquid $(\mathrm{a}-\mathrm{c})$. On the contrary, in situ water splitting can be used to generate a gas bubble that pushes the liquid out of the liquid cell and leaves an electrochemically active thin-film of liquid covering the sample, which allows obtaining near atomic-scale EDS analysis of a sample, which allows to perform high resolution analysis of an ongoing AuAg de-alloying process (d-f).

\section{References}

1. Kondratenko, E. V. et al., Energy Environ. Sci. 6, 3112-3135 (2013).

2. Ross, F. M. Opportunities and challenges in liquid cell electron microscopy. Science 350, 80, (2015).

3. Reimer, L. \& Kohl, H. Transmission Electron Microscopy: Physics of Image Formation. Springer, New York (2008).

4. Klein, K. et al., Microsc. Microanal. 17, 780-781 (2011).

5. de Jonge, N., Ultramic. 187, 113-125 (2018).

6. This work was performed in part at the Singh Center for Nanotechnology at the University of Pennsylvania, a member of the National Nanotechnology Coordinated Infrastructure (NNCI) network, which is supported by the National Science Foundation (NSF) (Grant NNCI-1542153). The authors gratefully acknowledge the use of facilities and instrumentation supported by the NSF through the University of Pennsylvania Materials Research Science and Engineering Center (MRSEC) (DMR1720530). 